\title{
On the use of Radio Environment Maps for Interference Management in Heterogeneous Networks
}

\author{
Jordi Pérez-Romero, Andreas Zalonis, Lila Boukhatem, Adrian Kliks, \\ Katerina Koutlia, Nikos Dimitriou, Reben Kurda
}

\begin{abstract}
This paper addresses the use of Radio Environment Maps (REMs) to support interference management optimization in heterogeneous networks composed of cells of different sizes and including both cellular and non-cellular (e.g., Wi-Fi) technologies. After presenting a general architecture for including REM databases in different network entities, the paper analyzes the achievable benefits in relation to specific interference management techniques, including a discussion on practical considerations such as information exchange requirements, REM ownership and security aspects. Finally, several research directions derived from the proposed framework are identified.
\end{abstract}

Keywords.- Radio Environment Map (REM), Interference Management, Heterogeneous Networks

\section{Introduction}

Recent years have witnessed an exponential growth in the demand for mobile broadband services associated with the massive penetration of mobile devices and the proliferation of bandwidthintensive applications. This trend is expected to continue to increase in the future with applications involving High Definition Video, virtual reality, etc. For the provisioning of such demanding services, the classical cellular network concept is being shifted toward Heterogeneous Networks (HetNets) [1] that combine large macrocells with smaller cells of different sizes. HetNets are expected to provide high capacities in densely populated areas and enhance coverage at specific locations (e.g., in indoor environments). They may also involve other access technologies, such as Wi-Fi, that can offload traffic from the cellular network. Although HetNets are already a reality in current Fourth Generation (4G) systems with the use of microcells, picocells and femtocells, they are expected to also be fundamental in future Fifth Generation (5G) systems that envisage extreme densification of small cells in certain scenarios.

Jordi Pérez-Romero and Katerina Koutlia are with the Dept. of Signal Theory and Communications at Universitat Politècnica de Catalunya (UPC). c/ Jordi Girona, 1-3, Campus Nord, D4 Building, Barcelona, Spain, 08034. e-mail: jorperez@tsc.upc.edu , katkoutlia@tsc.upc.edu.

Andreas Zalonis is with the Institute of Accelerating Systems and Applications, National Kapodistrian University of Athens, Greece. e-mail: azalonis@phys.uoa.gr

Lila Boukhatem and Reben Kurda are with LRI Lab., Univ. Paris-Sud 11 - CNRS, 91405 Orsay, France. e-mail: Lila.Boukhatem@lri.fr, Reben.Kurda@lri.fr

Adrian Kliks is with the Poznan University of Technology, Polanka 3, 60-965 Poznań, Poland. e-mail: akliks@et.put.poznan.pl

Nikos Dimitriou is with the Institute of Informatics and Telecommunications, National Center for Scientific Research "Demokritos”, Athens, Greece. e-mail: nikodim@iit.demokritos.gr

This work has been supported by FP7 NEWCOM\# project (grant number 318306) and by the Spanish Research Council and FEDER funds under RAMSES (ref. TEC2013-41698-R) grant. 
The widespread introduction of small cells requires enhanced Inter-Cell Interference Coordination (eICIC) methods for controlling and mitigating interference whenever the same frequency is shared by different cells. Various techniques have been developed and can be classified as frequencydomain techniques, time-domain techniques and power control techniques [2]. Frequency-domain techniques usually assign different frequency resources to users in the cells that can potentially cause interference [3]. In time-domain techniques, users suffering from interference are assigned resources in specific time periods where the interference is suppressed. Power control techniques adjust the transmitted power to reduce the interference generated to the victim users [4]. In addition, a proper user-to-cell association is essential to ensure that users are connected to the most convenient cell, and correspondingly, less interference is generated/received to/from the other cells. Moreover, when considering cellular and Wi-Fi networks (or other non-3GPP networks), it is also possible to reduce the interference in the cellular network by offloading traffic to the Wi-Fi network. This usually relies on the application of Access Network Discovery and Selection Function (ANDSF) and of the solutions proposed for so-called Hotspot 2.0 [5].

Given the randomness associated with propagation effects, user mobility, and traffic generation, the development of optimized eICIC techniques requires proper knowledge about the environment wherein HetNets are deployed. In this direction, the term Radio Environment Map (REM) refers to a database that dynamically stores information about the environment wherein a cognitive radio system operates [6]. It includes information about propagation conditions, locations of active transmitters in the area, traffic density, etc. This information can be exploited to optimize wireless networks, as in [7], where different applicability areas of the REM concept were identified.

Along these lines, this paper focuses on the use of REMs to support the optimization of eICIC for HetNets. In contrast to previous works, which have either identified different applicability areas of REMs or proposed specific algorithmic REM-based solutions to different problems, this paper intends to provide a comprehensive framework for how REMs can be used for interference management. For this purpose, the paper first addresses the architectural aspects associated with REMs. Then, it presents a detailed analysis of the benefits brought about by REMs in relation to specific eICIC techniques. The analysis includes a discussion of practical considerations such as information exchange requirements, REM ownership and security aspects. Finally, several research directions derived from the proposed framework are identified.

\section{REM architecture}

A REM functional architecture was proposed in [8], where the REM is composed of four main entities, namely, Measurement-Capable Devices (MCDs), which represent the network elements that perform measurements (e.g., terminals and sensors); a REM data Storage and Acquisition (REM SA) unit, which stores the data from the MCDs and the additional processed data; a REM manager, which requests measurements and extracts and processes the data from the REM SA; and a REM user as the entity using the REM data (e.g., a resource management entity or a policy manager).

The mapping of REM functionalities to specific network elements is closely associated with the architecture of the considered wireless network. For example, assuming a Third Generation Partnership Project (3GPP) Long Term Evolution (LTE) architecture with only femtocells, denoted as Home evolved Node Bs (HeNBs), [8] proposed that the REM SA functionality can be split into two parts: acquisition, included in the HeNBs, and storage, in the HeNB Management System (HeMS). In turn, the REM Manager functionality is associated with the HeMS [9]. In a more general topology that also includes macrocells, denoted as evolved Node Bs (eNBs), the architecture in [7] considers a layered REM composed of several instances of the same REM functional architecture located at different network nodes. This facilitates scalability because each REM entity only contains information about its local environment. Each layer may include only a subset of the REM functionalities, depending on the considered application. The upper level in the 
hierarchy is the REM entity at the network management subsystem of the operator. At lower levels, REM functionalities can be included in the Mobility Management Entity (MME), in the eNBs, in the HeNB GateWay (HeNB GW) or even in the User Equipments (UEs).

The REM concept can be extended for scenarios with various access technologies. The tight integration of cellular networks with Wi-Fi (or, more generally, the integration of 3GPP with non3GPP networks) is proposed as a meaningful solution to address high-load situations because it enables the offloading of some traffic from the cellular network, thus contributing to interference reduction. One approach toward this direction is the deployment of integrated Femto-Wi-Fi (IFW) modules [10]. In this context the REM may also include information about the Wi-Fi network (served users, positions, etc.), thereby allowing for efficient data offloading.

Taking as a reference the abovementioned approaches in an LTE heterogeneous cellular network with integrated Wi-Fi Access Points (APs), this paper identifies different architectural possibilities for implementing REM-based interference coordination. As the most general case, Figure 1 depicts a layered architecture whereby each eNB/HeNB contains a local REM entity with information about its local environment, and the MME and HeNB GW contain global REMs containing information at a large area level encompassing multiple eNBs/HeNBs. The global REM may include meta-information generated based on the fine-granularity data retrieved from the local REMs, which enables a macroscopic view and simplifies certain centralized decisions. Coordination between local REMs can be achieved through the X2 interface or through the global REM and S1 interface. With this layered approach, the local REM enables the support of localized resource management functionalities operating on short time scales, and an overall coordination at a lower rate can be achieved thanks to the global REM. This would be aligned with the current trend of shifting most of the decisions and operations as close as possible to the user, thereby contributing to latency reduction.

In the case of using integrated cellular and Wi-Fi networks for data offloading, communication between the REMs managing only cellular users and the REMs contained in the Wi-Fi APs or the IFW module can be realized through pure IP connections and/or S2a/S2c interfaces (see Figure 1).

Other possibilities can be derived from the architecture of Figure 1. One option would be a totally distributed architecture with only local REMs located at each eNB/HeNB and no global REMs. Coordination between REM instances to exchange information about neighboring cells could be achieved through the X2 interface. This solution may be efficient from the REM storage and management perspective because each REM only has to account for its local area. However, signaling associated with coordinating the different REM instances has to be considered as a function of the required information update rate, the amount of exchanged information, the computation and processing cost at each cell, etc.

Another option arising from Figure 1 would be a fully centralized approach with only global REMs at the MME and HeNB GW. Each REM will contain information about all of the cells in the controlled area. This will facilitate coordination but may involve large complexities and storage requirements if the number of cells is high. Moreover, REM-based decisions may be executed at a lower rate than in the distributed or layered case because of the latencies associated with accessing the REM. The coordination between the global REMs at MME and HeNB GW can be achieved through the S1 interface. Similarly, the possibility of having only the REM at the MME controlling both the eNBs and the HeNBs can also be considered, although this may not be efficient in the case of a very large deployment of HeNBs.

\section{REM-based Interference Coordination Techniques in HetNets}

This section analyses the use of REMs in relation to specific eICIC techniques, illustrates the benefits and discusses the practical and architectural implications. 


\subsection{REM-based elCIC Techniques}

The use of REM as a support tool in interference management in HetNets is applicable to a variety of different categories of eICIC techniques.

- Power control techniques:

These techniques adjust the transmit power of certain base stations of the network to reduce the generated interference. In a HetNet topology, with eNBs and HeNBs that serve a closed subscriber group of users, a critical challenge is the interference from an HeNB to nearby co-channel Macrocell User Equipments (MUEs). In this case, the transmit power of the HeNBs should be adjusted to avoid or reduce interference to the victim MUE. In [11], a baseline approach was presented, wherein each HeNB autonomously adjusts its transmit power based on its own received power measurements from the eNB. The introduction of a local REM in HeNB can enhance the effectiveness of this approach using the radio propagation characteristics of the surrounding area and the location of the neighboring MUEs, HeNBs and eNBs. This is demonstrated in the REMbased Autonomous HeNB Power Control (RAHPC) technique in which the HeNB uses the local REM to detect and locate the victim MUE and then it adjusts its transmission power to maintain a predefined Signal-to-Interference-and-Noise Ratio (SINR) target for the MUE [12]. Another proposal is REM-based Macrocell-Assisted Power Control (RMAPC), where the eNBs support the power adjustment of HeNBs by considering the contribution of each HeNB to the total interference and the impact on the outage of both MUEs and HeNB User Equipments (HUEs) [13]. In this case, the HeNBs' local parameters are stored in the REM, and the eNB can use them to achieve the globally coordinated power adjustment.

- Frequency domain techniques:

In this category, the REM can support the optimal selection of sub-bands to be used in the macrocells and small cells, therein targeting the minimization of inter-cell interference. One approach is the Gibbs sampler-based technique originally proposed in [14] in the context of macrocell scenarios. Here, it is extended to a HetNet scenario by also considering its implementation based on the REM concept. In this technique, which is denoted in the following as REM-based Frequency Optimization (RFO), small cells use a single sub-band, while eNBs use two different sub-bands, one for the users located in the inner part of the cell and the other for the users located in the outer part of the cell. Then, at random instants defined by an exponential timer, each cell modifies the used sub-bands following a Gibbs-Boltzmann distribution that selects with higher probability those sub-bands where the cell receives and generates less interference to their neighbor cells. This is performed iteratively so that the system progressively reduces the total inter-cell interference. The estimation of the received and generated interference is performed based on the propagation losses between users and neighbor cells that are obtained from the REM.

- Time-domain techniques:

REM-related information can also be helpful in developing an optimal configuration of the muting periods of the macrocells, i.e., Almost Blank Subframes (ABS) [2], to enable interference-free small cell transmission. REM will help in the identification of small-cell users that are more sensitive to macrocell interference and in deciding how many ABSs are needed.

- Wi-Fi offloading:

Efficient data offloading from the cellular network via the Wi-Fi network (or, in general, via other non-3GPP networks) allows a decrease in the HeNB/eNB load, and consequently, it can simplify the interference management in the cellular network [15]. REM can be considered as a technical enabler for this offloading because it can contain information about the detailed locations of available Wi-Fi networks and their characteristics. 


\subsection{Architectural Considerations}

The selection of an eICIC technique impacts the choices derived from the architecture of Figure 1 concerning the use of the local/global REMs or the type of stored information. The architectural considerations of the techniques described in the previous sub-section are presented in the following, and Table 1 lists the specific REM information used in each case.

- Local REM information:

The more dynamic parameters of the radio environment and the information that only affects a reduced number of nodes will be preferably stored in the local REMs because this would facilitate the REM updates. As shown in the examples in Table 1, this mainly includes radio-propagationrelated information, such as propagation losses, signal strengths, and the locations of certain nodes such as mobile terminals or HeNBs.

- Global REM information:

Usually, global REMs will store the less dynamic parameters or the parameters that may affect a high number of network nodes. As observed in Table 1, the stored information includes certain Quality of Service (QoS) metrics, the positions of eNBs and information related to available Wi-Fi access networks such as the ownership (e.g., private or public, fee-based access or free access, and with or without authorization), the quality of the IP addressing options and the parameters of the available backhauling options for each Wi-Fi.

- Mapping of REM entities:

In all of the strategies considered here, the local REM of each cell includes the REM manager and REM SA, and MCDs will be the mobile terminals and the cells, whose measurements will be used to build the REM data.

\subsection{Benefits}

The use of a REM in the abovementioned techniques improves the HetNet performance in terms of various metrics.

- Capacity and throughput improvement:

The information stored in the REMs helps increase the cell capacity and/or user throughput. To illustrate this, two examples are discussed. First, Figure 2 depicts the throughput gains that are obtained for MUEs with the RMAPC strategy in relation to a baseline solution where no REMbased power control is applied. Two versions of the RMAPC mechanism are considered, which differ in their selection strategy of the set of HeNBs used to execute the power adjustment and the amount of power to reduce. The first version is more aware of HUEs' performance degradation, and the second version prioritizes MUEs. Gains of $18 \%$ for MUEs are observed at the cost of a slight degradation of the HUEs' throughput, which remains under their minimum requirements. Concerning the RFO technique, Figure 3 presents the capacity increase with respect to a reference scheme that assumes that eNBs follow a classical Fractional Frequency Reuse and that the sub-band allocated to a small cell is randomly selected among those not used by the closest eNB. Figure 3 shows that, for the ideal case without errors where the REM information matches the real propagation losses (i.e., $\varepsilon=0 \mathrm{~dB}$ in the figure), very significant capacity gains of between 30 and $55 \%$ for small-cell users, depending on the inner cell radius of the eNBs, were observed with negligible impact on the capacity of MUEs.

- MUE outage reduction:

In the RAHPC technique, the use of REM can significantly reduce the MUE outage (i.e., the probability of being below the SINR target) with respect to the baseline scheme of [11]. This is illustrated in Figure 4 in a specific scenario with a transmitting co-channel HeNB in close proximity to an MUE [12]. The biggest improvement is observed for the case when the victim MUE is located 
close to the eNB so that it receives a strong signal from this eNB. In this case, the outage is reduced by approximately $15 \%$ in the presented scenario. In this respect, it is envisaged that this type of technique can be useful in future scenarios with extreme densification of cells in certain areas, in which the situations with low signal strength will be reduced and the main challenge will be in the interference control.

\section{- Traffic offloading:}

The use of REM in Wi-Fi/cellular scenarios can lead to an efficient traffic offloading from the cellular network to the Wi-Fi network, which in turn simplifies the interference management in the cellular network. In particular, it is shown in [15] that up to $30 \%$ of the total traffic can be shifted to the Wi-Fi network without violating the QoS, assuming that the REM has perfect knowledge about current Wi-Fi channel utilization, Wi-Fi APs and small-cell base-stations locations.

\subsection{Practical aspects}

Interference management techniques compel the REM manager to obtain fast and reliable access to information from various sources (e.g., from cellular or other non-3GPP network elements or from dedicated sensor networks). Depending on the scenario and the applied algorithms, both dynamic and static information can be considered. The singularities of each case will dictate the best practical approach, thereby attempting to balance the accuracy of the utilized information, latency issues, processing complexity, and related management and security aspects. This section analyzes some of these practical considerations.

\section{- Information exchange:}

Because interference optimization techniques usually require short-time-scale interactions, direct interfaces, such as $\mathrm{X} 2$, enable fast data exchange between local REM entities. Signaling requirements will depend on the utilized technique. For example, in the RFO approach, each time the algorithm is executed, the local REM of a cell needs to receive information from its neighbor cells, including the propagation losses to the users of these cells and the transmit power in each subband. Let us assume that the propagation loss for a user to a cell is encoded with $L_{P}$ bits, the transmit power per sub-band is encoded with $L_{T}$ bits, and the header of the REM message has $L_{H}$ bits. Then, for a total of $N_{\text {bands }}$ sub-bands, $N_{U}$ users per cell, $N_{\text {neigh }}$ neighbor cells and a decision procedure executed on average every $t_{a} \mathrm{~s}$, the local REM signaling requirements per cell will be $\left(L_{H}\right.$ $\left.+N_{u} \cdot L_{P}+N_{\text {bands }} \cdot L_{T}\right) \cdot N_{\text {neigh }} / t_{a}$ (bits/s). In the scenario considered in Figure 3, assuming $L_{H}=16$, $L_{P}=7, L_{T}=4, N_{\text {neigh }}=20, N_{\text {bands }}=4, N_{u}=10$, and $t_{a}=30 \mathrm{~s}$, the REM signaling requirement is $68 \mathrm{bits} / \mathrm{s}$ per cell, which can be considered a quite acceptable value. In the case of more centralized solutions involving global REMs, solutions should be developed under the premise that only local databases are updated frequently, whereas information about global REMs is updated at a lower rate. This will reduce the signaling traffic in the backhaul links.

- Building REM information:

Various methods can be used to collect REM-related information. In feedback-based mechanisms, the network elements (eNBs, HeNBs, and terminals) collect/measure and report information related to channel gains, location, sub-band use, etc. However, additional mechanisms may be needed for certain techniques, such as the RAHPC approach, where the local REM in HeNB should estimate the location of victim MUEs that do not communicate their location to the HeNB. In this case, the incorporation of sensing capabilities in the HeNBs or the use of a dedicated sensor network in the HeNB vicinity should be considered.

- Robustness against errors:

The abovementioned REM building process will impact the reliability of the stored information, which in turn will influence the performance achieved by a REM-based interference management technique. To illustrate this point, Figure 3 presents the capacity gain achieved by the FCO 
technique as a function of the error in the propagation losses stored in the REM. For each value, the error is modeled as a uniformly distributed random variable in the range $[-\varepsilon, \varepsilon] \mathrm{dB}$. It is observed that, as the REM error increases, the capacity improvements are progressively reduced, although continuing to maintain significant values, revealing the robustness against errors in the considered approach.

- REM ownership and management:

In the context of interference management, a natural approach is that the REM is owned and managed by the cellular network operator, who will have complete control over the REM functionalities. In the case of multi-operated HetNets, such as when a Wi-Fi network belongs to a different provider than the cellular operator, or when different cellular operators cooperate for interference coordination purposes (e.g., when shared or unlicensed spectrum is used), three possible solutions can be identified for REM management and ownership. The first option is that each operator possesses its own databases, and a dedicated and secured protocol is used for data exchange among 3GPP and non-3GPP networks ${ }^{1}$. In this case, a REM user will have access to the REMs of its operator but will also indirectly benefit from the local and global REMs of cooperating operators. A second option is a hybrid solution whereby some operators decide to merge their REM databases or apply techniques for transparent data sharing. Finally, another option is the establishment of a third-party dedicated provider responsible for REM construction and management. This solution does not exclude the existence of local and global REMs by each operator.

- Security and privacy:

From the user perspective, because the REM databases may store sensitive information for interference coordination purposes, security and privacy constitute significant challenges. Privacy threats against personal information, such as fine-grained user locations, should be addressed in order not to disclose this information against the users' will. When the REM owner is the operator, the REM should be accessible only from entities residing within the network operator itself, which will ensure that the REM contents will have a similar level of security than other elements of the operator network. Then, users' data integrity and confidentiality can be guaranteed at the same level as the private data of all the mobile users. The same situation occurs for data that the operator does not want to disclose to others. In the hybrid solution whereby some operators merge their databases, information exchange with non-3GPP networks can be realized by dedicated secured protocols (e.g., IPSec), but again, the ownership and security assurances remain under the auspices of the network operators. In that sense, an extension of existing intra-network security solutions could be envisioned for the secure access of the REM. In the case where the interconnecting databases are under the management of a third-party entity, additional security mechanisms should be designed. Therefore, the relevant regulatory bodies and the operators must determine the best approach to protect the information.

\section{Research directions}

The analysis of previous sections paves the way for further research directions related to REM applicability for interference management in HetNets. In this respect, the following main directions are identified:

- More sophisticated eICIC techniques can be developed by combining the dimensions of power, frequency, time and user-to-cell association into a multi-parameter optimization framework supported by REMs. REM information will allow the identification of users that are more sensitive

\footnotetext{
${ }^{1}$ For example, the Next Generation Hotspot certified with Wi-Fi Certified Passpoint ${ }^{\mathrm{TM}}$, from which rich information on the Wi-Fi network can be obtained.
} 
to interference in each situation and adjustment of different parameters accordingly. For example, the adequate number of ABSs should be decided to properly protect these users while simultaneously not excessively degrading the performance of the remaining users of the macrocells. Similarly, depending on the characteristics of the scenario, it may be more convenient to allow transmission at a reduced power in certain subframes instead of completely muting the transmission during ABSs. In addition, some subframes can be configured by reserving certain resources in the frequency domain for users located in certain areas of the cell (e.g., the small-cell users located at the edge). These combined techniques require setting multiple parameters (e.g., number of ABSs, transmit powers, and fraction of reserved resources); thus, their optimization requires accurate knowledge of the conditions that each user/cell is experiencing to properly assess the impact of the variations in each parameter on the achieved performance. Specific challenges in this context include the placement of the optimization algorithms, the representation of the REM parameters, the update rate, and the accuracy of the stored information.

- Interference management will be a key component in scenarios with extreme densification of cells in certain areas (e.g., malls and stadiums). In these scenarios, a terminal may receive high signal levels from a very large number of cells, thus requiring more efficient solutions to interference coordination and user-to-cell association. In such complex environments, the multi-layered REM will address the optimization of interference coordination in a simpler manner and facilitate scalability, e.g., by clustering the local REMs of cells with stronger interactions under the control of a global REM.

- Novel architectural paradigms, such as Network Function Virtualization (NFV) and Software Defined Networking (SDN), are envisaged to facilitate the introduction of the REM concept. NFV refers to the software implementation of network functions running on general purpose computing/storage resources. This can be applied to radio access in the form of a cloud-Radio Access Network (C-RAN). SDN refers to decoupling network control and data planes, thereby enabling the implementation of control functions as software applications running on top of an SDN controller that provides a programmatic interface to the network. Through SDN/NFV, local/global REMs could be implemented as virtual databases supporting the interference control functions implemented as software packages. In this manner, the system becomes substantially more adaptive and flexible and can be used to dynamically optimize the information split between local/global REMs and quickly introduce new stored parameters as needed by the interference control functions.

- Further challenges arise in the evolution toward heterogeneous 5G systems involving multiple technologies, a wider range of spectrum bands (e.g., millimeter waves and unlicensed bands), additional types of wireless links (e.g., backhaul and Device-to-Device) and new interworking challenges. REMs may support advanced optimization algorithms for interference coordination, efficient sharing of unlicensed bands, energy-efficient user-to-cell associations as well as backhauling aspects, e.g., to identify/predict the existence of a line-of-sight link between access points. The vision of a converged network, or network of networks with close cooperation between them, can also be facilitated through REMs that provide the contextual information to make decisions affecting such cooperation.

\section{Conclusions}

This paper has proposed the use of REMs for supporting interference management in HetNets. A general layered architecture including global and local REM databases in the context of a network with both cellular and Wi-Fi technologies has been suggested. The benefits and architectural implications of REMs have been illustrated for various specific techniques, and we have discussed the achievable gains in terms of capacity or outage reduction, including considerations of ownership and security. The paper has concluded with a list of research challenges derived from the presented framework. 


\section{References}

[1] A. Ghosh, N. Mangalvedhe, R. Ratasuk, B. Mondal, M. Cudak, E. Visotsky, T.A. Thomas, J.G. Andrews, P. Xia, H.S. Jo, H.S. Dhillon, T. D. Novlan, "Heterogeneous Cellular Networks: From Theory to Practice,” IEEE Communications Magazine, vol. 50, no. 6, June, 2012, pp. 54-64.

[2] D. Lopez-Perez, I. Guvenc, G. De la Roche, M. Kountouris, T.Q.S. Quek, Jie Zhang, "Enhanced intercell interference coordination challenges in heterogeneous networks," IEEE Wireless Communications, vol. 18, no. 3, June 2011, pp. 22-30.

[3] T. Novlan, R. Ganti, A. Ghosh, J. Andrews, "Analytical Evaluation of Fractional Frequency Reuse for OFDMA Cellular Networks,”, IEEE Transactions on Wireless Communications, vol. 10, no. 12, December, 2011, pp. 4294-4305.

[4] 3GPP R1-103823, "HeNB Power Setting Performance under Different Access Constraints," July, 2010.

[5] B. Orlandi, F. Scahill, "WiFi-Roaming - Building on ANDSF and Hotspot 2.0", White paper of Alcatel-Lucent and BT, 2012, available at http://www.tmcnet.com/tmc/whitepapers/documents/whitepapers/2013/6686-wi-fi-roamingbuilding-andsfand-hotspot20.pdf, accessed 29th April 2015.

[6] Y. Zhao, L. Morales, J. Gaeddert, K. K. Bae, J.-S. Um, and J. H. Reed, “Applying radio environment maps to cognitive wireless regional area networks,” 2nd IEEE International Symposium on New Frontiers in Dynamic Spectrum Access Networks (DySPAN 2007), Dublin, Ireland, April, 2007.

[7] J. van de Beek, T. Cai, S. Grimoud, B. Sayrac, P. Mähönen, J. Nasreddine, J. Riihijärvi, "How a Layered REM Architecture Brings Cognition to Today’s Mobile Networks,” IEEE Wireless Communications, vol. 19, no. 4, August, 2012, pp. 17-24.

[8] B. Sayrac (editor), "D2.4: Final System Architecture,” Deliverable of FARAMIR project, December, 2011, available at http://www.ictfaramir.eu/fileadmin/user_upload/deliverables/FARAMIR-D2.4-Final.pdf, accessed 29th April 2015.

[9] 3GPP TS 36.300 v11.7.0 “Evolved Universal Terrestrial Radio Access (E-UTRA) and Evolved Universal Terrestrial Radio Access Network (E-UTRAN); Overall description; Stage 2 (Release 11),” September, 2013.

[10] SmallCellForum, “Integrated Femto-WiFi (IFW) Networks”, Document Number 033.05.01, December, 2013.

[11] Femtoforum, “Interference Management in OFDMA Femtocells,” March, 2010.

[12] A. Zalonis, N. Dimitriou, A. Polydoros, J, Nasreddine, P. Mähönen, "Femtocell Downlink Power Control based on Radio Environment Maps," IEEE Wireless Communications and Networking Conference (WCNC), Paris, France, April 2012.

[13] R. Kurda, L. Boukhatem, T. Ali-Yahiya, M. Kaneko, "Power adjustment mechanism using context information for interference mitigation in two-tier heterogeneous networks," IEEE $19^{\text {th }}$ Symposium on Computers and Communication (ISCC), Madeira, Portugal, June 2014.

[14] K. Koutlia, J. Pérez-Romero, R. Agustí, M. Ziak, “On the use of Gibbs Sampling for InterCell Interference Mitigation under Partial Frequency Reuse Schemes," The Third International Conference on Mobile Services, Resources, and Users (MOBILITY), Lisbon, Portugal, November, 2013. 
[15] A. Kliks, A. Zalonis, N. Dimitrou, O.Holland, "WiFi Traffic Offloading for Energy Saving," 20th International Conference on Telecommunications (ICT), Casablanca, Morocco, 6-8 May, 2013. 


\section{Biographies}

Jordi Perez-Romero (jorperez@tsc.upc.edu) is associate professor at the Dept. of Signal Theory and Communications of the Universitat Politecnica de Catalunya (UPC) in Barcelona, Spain, where he received the Telecommunications Engineering and the PhD degrees in 1997 and 2001, respectively. His research interests are in the field of mobile communication systems, especially radio resource and QoS management, heterogeneous wireless networks and cognitive networks. He has been involved in different European Projects and in projects for private companies.

Andreas Zalonis has a B.Sc. degree in Physics (2000) and a M.Sc. degree in Telecommunications and Electronics (2002), both from National Kapodistrian University of Athens (NKUA), Greece. Since 2003 he works as a Research Associate at the Institute of Accelerating Systems and Applications (IASA) at NKUA. He has participated in several research projects; his main research interests are radio resource management and flexible/adaptive transceiver design for wireless communication systems.

Lila Boukhatem (Lila@lri.fr) is associate professor at LRI laboratory - University of Paris-Sud 11. She received M.Sc degree in 1998 from the University of Versailles (UVSQ) and Ph.D. degree in 2001 from the University of Paris 6. She joined the LRI laboratory in 2002 where she is developing various research works in mobile and wireless networks including: Cross-layer design, modeling and performance evaluation, resource allocation, interference mitigation, mobility management, routing and energy in ad hoc, vehicular and sensor networks. She was involved in several national and international projects and programs.

Adrian Kliks (akliks@et.put.poznan.pl) received with honors M.Sc. and Ph.D. degrees in telecommunications from Poznan University of Technology (PUT), Poland, in 2005 and 2011, respectively, and currently he is employed as assistant professor at the Chair of Wireless Communications, PUT. He was involved in various industrial and international research projects. His scientific interests include i.a. advanced multicarrier communications, waveform design, smallcells in heterogeneous networks, WiFi offloading, and cognitive radio.

Katerina Koutlia received her BA in Electronics Engineering (2009) from the Technological Institution of Thessaloniki (Greece) and her MSc with distinction (2011) in Wireless Communication Systems from the Brunel University (Uxbridge UK). Currently she is a PhD student in the Mobile Communication Research Group (GRCM) at the Polytechnic University of Catalonia (UPC). Her current research interests include Inter-cell Interference Coordination Techniques (ICIC), Resource Management and Heterogeneous Networks.

Nikos Dimitriou (SM.IEEE, 2011) holds a Diploma in Electrical \& Computer Engineering from the National Technical University of Athens, Greece (1996), an M.Sc. with distinction in Mobile and Satellite communications (1997) from the University of Surrey, UK and a Ph.D. in Mobile Communications from the same university (2001). He is currently a Research Fellow in the Institute of Informatics and Telecommunications in the National Center of Scientific Research "Demokritos". His research interests include Radio Resource Management for dense HetNets and Robust Routing for Mobile Ad-Hoc Networks.

Reben Kurda earned the bachelor's degree (BA) in computer science from the University of Sulaymaniyah, Kurdistan-Iraq (2003) and the M.Sc. degree in informatics of the University of Koya, Kurdistan-Iraq (2007). In 2015, he obtained his Ph.D degree in wireless communications from the University of Paris-Sud 11, France. He is currently employed as a lecturer at the University of Koya, Kurdistan-Iraq. He was involved in various industrial and international research projects. His scientific interests include LTE-Advanced multicarrier communications, interference mitigation in and small cell and heterogeneous networks, and Wi-Fi offloading. 


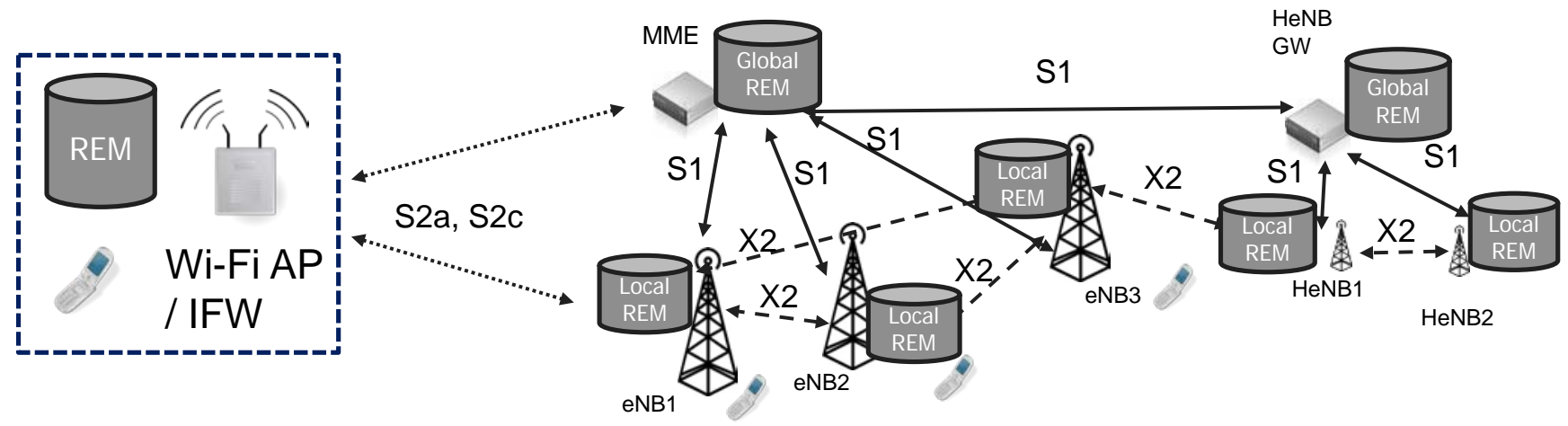

Figure 1. Layered REM architecture 


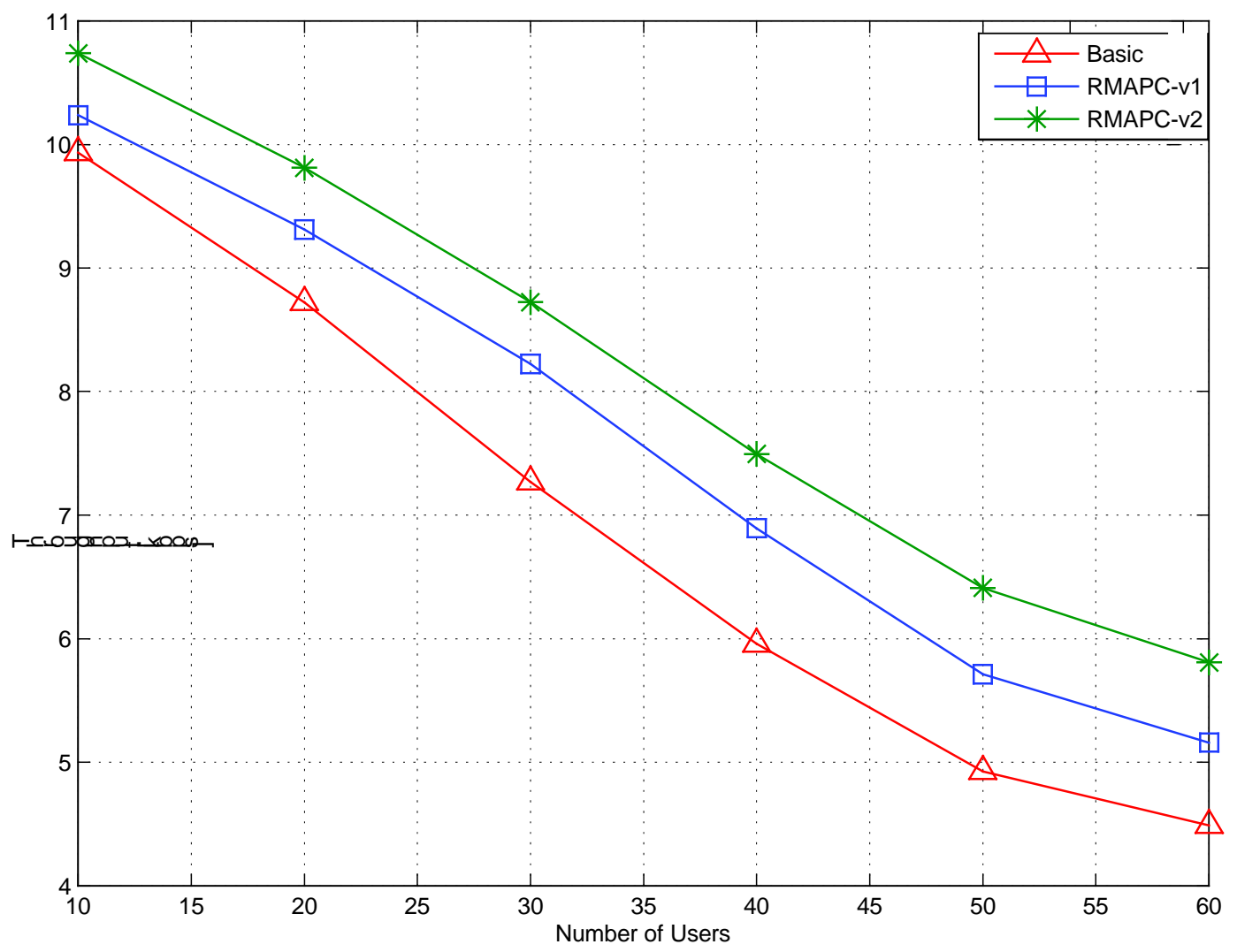

Figure 2. Throughput gain for macrocell users for Constant Bit Rate flows with the RMAPC technique 


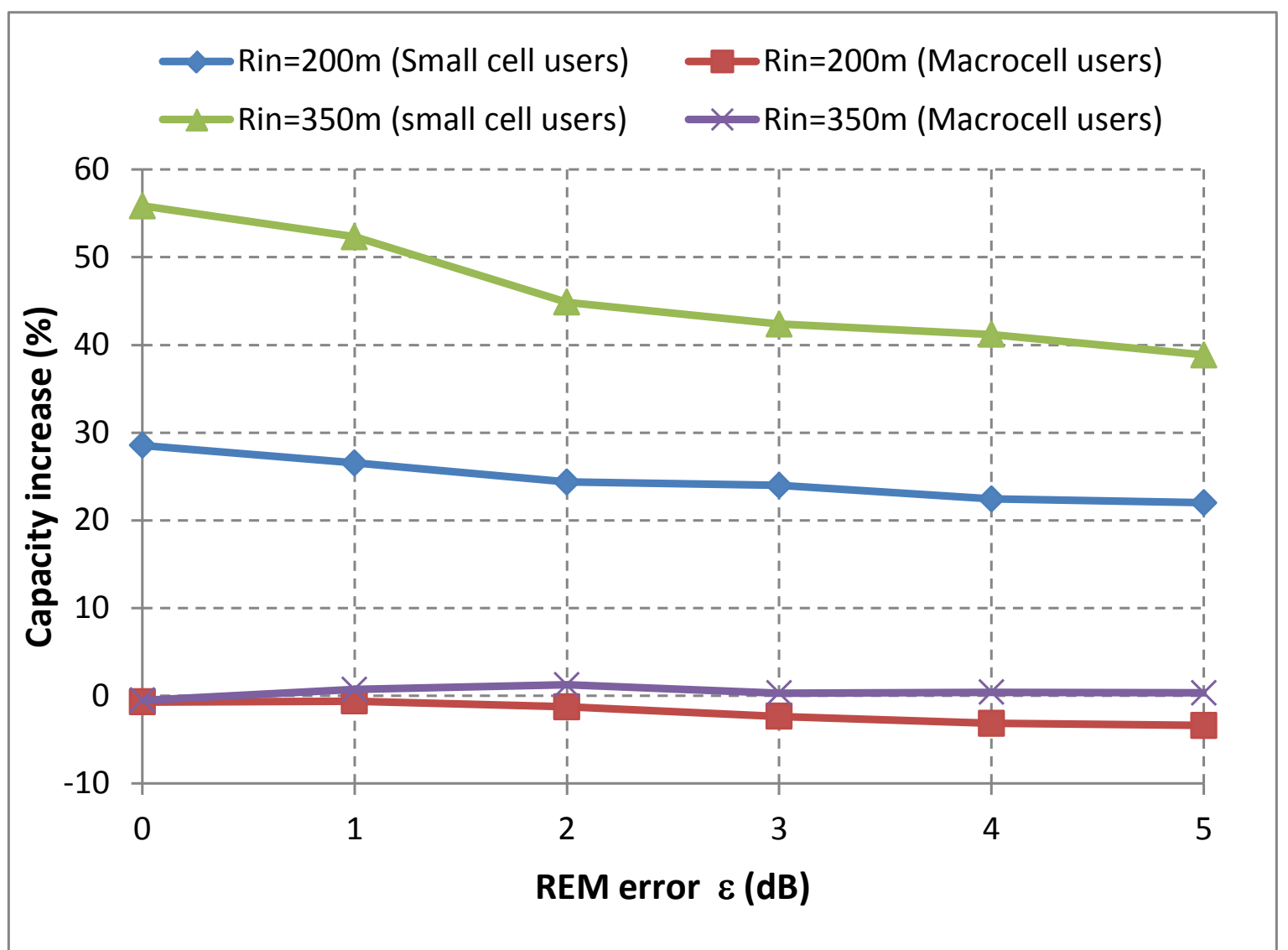

Figure 3. Increase in the downlink average capacity per user achieved by the RFO technique for different values of the REM information error $\varepsilon$. The results are presented for a scenario with $12 \mathrm{eNBs}$ and 8 small cells with radius $500 \mathrm{~m}$ and $100 \mathrm{~m}$, respectively.

Two values of the eNB inner cell radius Rin are considered, namely, 200 and $350 \mathrm{~m}$, corresponding to transmit powers of the inner part of 28 and $37 \mathrm{dBm}$, respectively. The transmit power for the outer users is $43 \mathrm{dBm}$, and the transmit power of the small cells is $20 \mathrm{dBm}$. The propagation models are from [14]. 


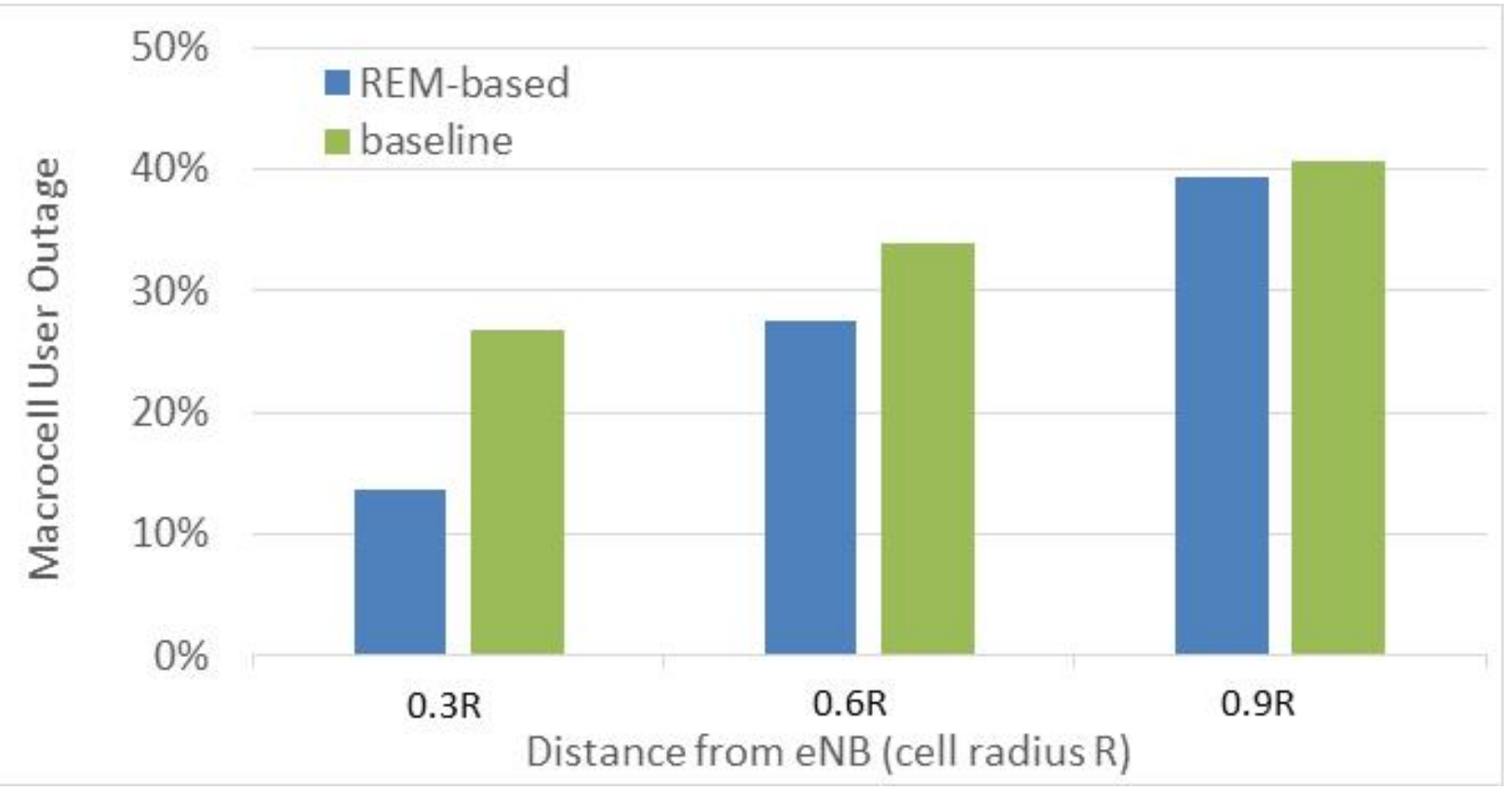

Figure 4. Average MUE outage for various distances between the eNB and the MUE (eNB radius $R=1 \mathrm{~km})$. The MUE is placed in a random position close to a co-channel HeNB (inside or outside the house) with a SINR target of 3dB in a suburban scenario. Details of simulation parameters can be found in [12]. 
Table 1.- Categorization of the parameters in the Local/global REM databases and how they are used in the different strategies

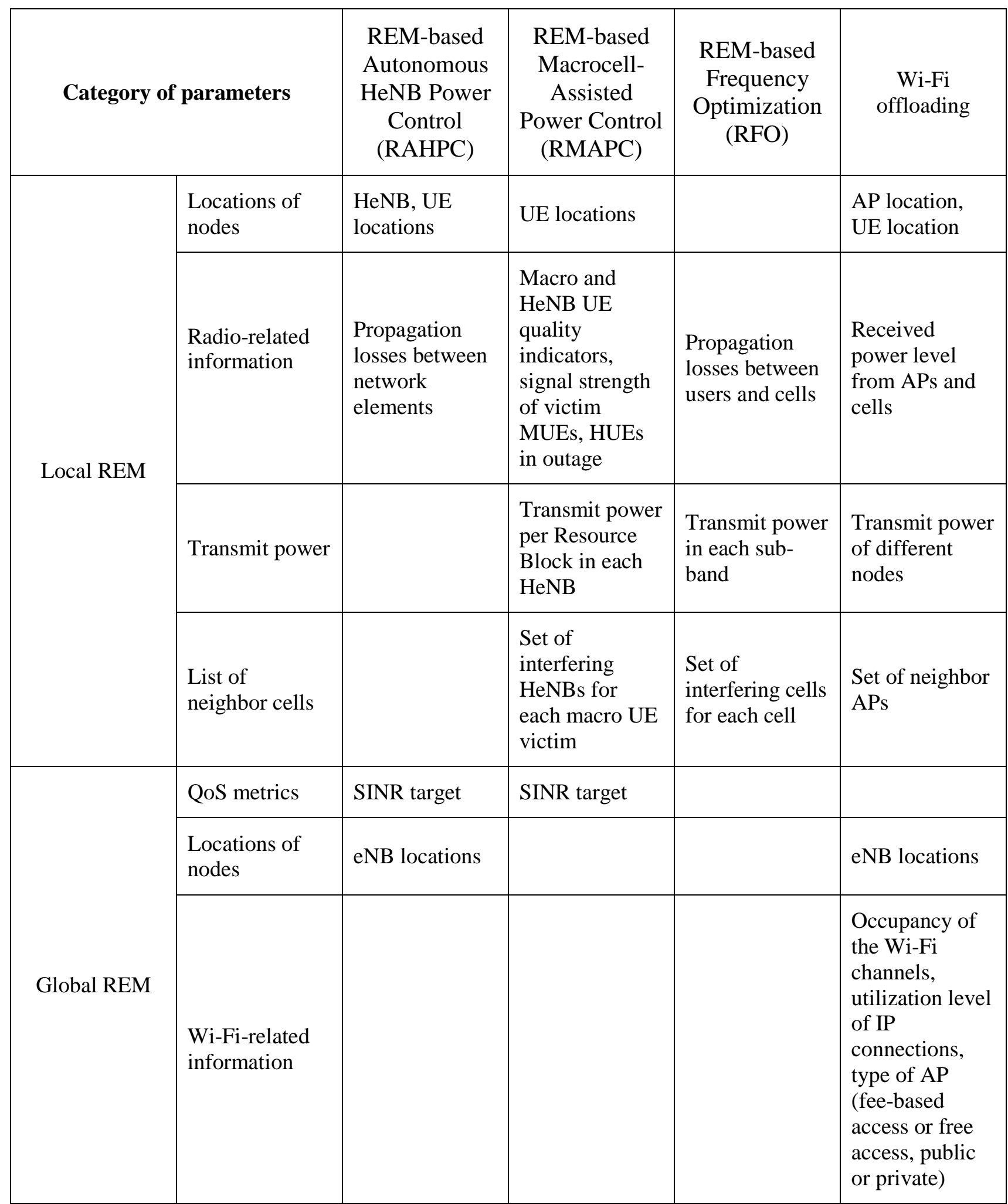

